\title{
Linx
}

Revue des linguistes de l'université Paris X Nanterre

$80 \mid 2020$

L'héritage de Jean Dubois et Françoise Dubois-Charlier

\section{Forger des phrases simples dans un dictionnaire de langue générale}

Les Verbes français : un exemple à partir des emplois figurés.

Les verbes français: a metalexicographic approach of examplification through simple sentences

\section{Antoinette Balibar-Mrabti}

\section{OpenEdition}

\section{Journals}

Édition électronique

URL : http://journals.openedition.org/linx/6627

ISSN : 2118-9692

Éditeur

Presses universitaires de Paris Nanterre

Référence électronique

Antoinette Balibar-Mrabti, «Forger des phrases simples dans un dictionnaire de langue générale », Linx [En ligne], 80 | 2020, mis en ligne le 10 juillet 2020, consulté le 05 août 2020. URL : http:// journals.openedition.org/linx/6627

Ce document a été généré automatiquement le 5 août 2020

Département de Sciences du langage, Université Paris Ouest 


\section{Forger des phrases simples dans un dictionnaire de langue générale}

Les Verbes français : un exemple à partir des emplois figurés. Les verbes français: a metalexicographic approach of examplification through simple sentences

Antoinette Balibar-Mrabti

\section{Une mise en perspective historique d'ordre métalexicographique}

1 Comment faire des choix d'illustration systématique dans un dictionnaire de langue dès lors qu'on se place à la marge des sens propres, en énumérant et classant des emplois de verbes reconnus comme étant détournés et qualifiés, à ce titre, dans la terminologie usuelle, comme étant imagés ou figurés ? Mes observations concernent le travail préparatoire à la rédaction des Verbes français ${ }^{1}$ (désormais LVF 1997) sur ce que les auteurs appellent de façon traditionnelle, en s'adossant aux manuels scolaires les plus courants, des phrases simples (LVF 1997 : VIII).

Quelle est la définition d'une phrase simple? Un examen de tables des matières de manuels met en évidence un emploi récurrent du terme ${ }^{2}$, depuis les grammaires les plus élémentaires, parmi lesquelles figure une grammaire de Jean Dubois lui-même $L a$ nouvelle grammaire du français, (Dubois et Lagane 1973), jusqu'aux plus complètes de sa génération. Citons Le Bon usage (1936-1980) de Maurice Grevisse continué et réédité par André Goose jusqu'en 2016, la Grammaire Larousse du Français Contemporain (Chevalier et al. 1964), que renouvelle La Grammaire méthodique du français (Riegel et al. 1994) ainsi qu'une refonte du manuel de Grevisse et Goose avec Le Petit Bon usage de la langue française (Fairon et Simon 2018). Le plus intéressant est de remarquer le contraste suivant avec deux cas types qui pourraient surprendre. Tout d'abord la phrase dite simple s'utilise uniquement comme rubrique, sans autre précision, quand elle se trouve dans un des ouvrages les plus précis et les plus élaborés, destiné aux utilisateurs 
instruits, Le Petit Bon usage ${ }^{3}$. Elle n'en est pas moins abondamment requise à travers la recherche systématique d'exemples mis à jour qui sont tirés d'écrivains francophones reconnus. Cette pratique rejoint directement celles des lexicographes et j'y reviens en sections 2 et 3. En contraste, les indications parmi les plus claires se trouvent dans $L a$ nouvelle grammaire du français de Jean Dubois (pp. 151-154), rédigée pour un niveau d'instruction moins ambitieux qui correspond aux écoliers et aux collégiens actuels. On y détaille un certain nombre de manipulations autour de la notion de "phrase étendue " qui se distingue de la phrase complexe, traditionnellement opposée à la phrase simple à partir de l'enseignement du latin. Dans cette ligne, le Grevisse de l'enseignant (Pellat et Fonvielle 2016) est le manuel récent le plus pertinent.

Dans tous les cas il existe un implicite partagé, plus littéraire que grammatical ${ }^{4}$. Ce nondit mériterait une étude à part entière, tournée vers le rapport grammaire/littérature crucial en philologie et relayé ici en pédagogie. On le retrouve en français numérisé avec les méthodes de simplification automatique des textes ou SAT (Gala 2018 :123-131). Précisons d'entrée de jeu que Jean Dubois, quand il fait le bilan de ses travaux (Chevalier et Encrevé 2006) prend du recul vis-à-vis des polémiques de sa génération liées à l'introduction de la grammaire structurale américaine et indique clairement que la grammaire et philologie lui a apporté et nous apporte encore des dimensions d'étude scientifiques des langues. Une évidence pour les historiens des idées linguistiques et surtout ici un indice de sa culture littéraire des textes, véhiculée à son époque à travers Ferdinand Brunot et prolongée par Charles Bruneau soucieux des styles, tous deux descendus de leur piédestal académique dans sa génération de grammairien qui fut aussi celle de Jean-Claude Chevalier, initiateur en France de l'histoire de la syntaxe. J'y reviens en section 2 .

Dans la ligne de ma contribution "Exemples lexicographiques et métaphores" (Balibar-Mrabti 2002, pp. 90-108) je poursuis ici une réflexion sur normes et dictionnaires de langue générale. Avec quelle culture grammaticale incluant notre héritage en matière de didactique et de "lexiques régulés " (Chevalier 1997 ; 1998) ? Par quelles techniques de l'ordre de la linguistique appliquée, quand on opère un passage des compilations lexicales vers les outils de consultation beaucoup plus élaborés que sont nos dictionnaires dits de langue française avec leurs formes actuelles tournées vers le numérique (Gala et Zock 2013) ? Envisagées en durée longue d'histoire des idées linguistiques, les techniques mises en jeu se rattachent à l'histoire générale de l'inscription des langues sur support écrit. Elles obéissent à une " raison graphique » (Goody 1979) également qualifiée de "grammatisation » (Auroux 1995) où prend place précisément la syntaxe, comme vecteur de classification valorisé avec LVF.

5 Sur la question ici des dédoublements d'entrées de dictionnaires papiers et/ou électroniques, dont relève LVF pour traiter des emplois métaphoriques de verbes, je renvoie aux clarifications, d'ordre purement lexicographique, que j'avais demandées à Christian Leclère (2002) quand j'ai dirigé Langue Française 134. Le programme de Jean Dubois s'apparente étroitement aux recherches du Laboratoire d'Automatique Documentaire et Linguistique (LADL) impulsées par Maurice Gross. Le LADL, rappelonsle, hébergea dans ses locaux l'équipe des rédacteurs de LVF dont on sait précisément qu'une large part d'entre eux menaient en parallèle ces types de recherches, centrées sur une visée pionnière de modernisations des dictionnaires, distinctes mais apparentées. On soulignera ici la mise en commun, chez tous ces chercheurs, de la notion préthéorique intuitivement partagée, à valeur exploratoire, de phrase simple ${ }^{5}$ 
comme matériau initial à classer. J'en ferai ici le point de départ de mes remarques qui sont d'ordre métalexicographique.

6 Comment opérer avec LVF une transition raisonnée ${ }^{6}$ entre un type de dictionnaire électronique, encore largement à inventer, et les dictionnaires papiers de tous types du $20^{\text {ème }}$ siècle dont les Dubois étaient précisément des rédacteurs de premier plan ? Côté dictionnaires papier du français contemporain, notamment dans la riche production des dictionnaires élémentaires, Jean Dubois, avec Françoise Dubois-Charlier succédant à Alain Guillet, auront mené un travail largement comparable, par les objectifs et les savoir-faire, à celui d'Alain Rey et Josette Rey-Debove dirigeant la série des dictionnaires Robert. De fait, c'est la réunion des Robert et des Larousse que donnent Jean-Paul Boons, Alain Guillet et Christian Leclère (1976 et 1992, ouvr. cit.) dès qu'ils précisent leurs sources documentaires. Côté traitement automatique, l'entreprise de Jean Dubois, et sa durée d'exécution, ne s'apparente pas seulement aux classifications du LADL. Ses principes d'exécution gagnent en clarté quand ils sont comparés et évalués en fonction des résultats présentés par le Dictionnaire explicatif et combinatoire (DEC) d'Igor Mel'čuk. D'ailleurs le succès du DEC a souvent fait écran à LVF dans l'immédiate actualité des travaux de recherche. Les deux auteurs héritent des classifications du LADL initiées par Maurice Gross avec Méthodes en syntaxe (1975) dont ils proposent, chacun à leur manière, des remaniements débouchant sur deux résultats autonomes de classification des mots, considérés à l'échelle d'un lexique pris désormais en taille dite « réelle ». Dans les deux cas, intervient une exigence jugée incontournable durant les deux décennies suivantes (années 1980-1990) : introduire explicitement ${ }^{7}$ des calculs du sens.

7 Concernant mon examen des tenants et aboutissants choisis par Jean Dubois pour la mise en œuvre de LVF, je me distancie ici de tout débat direct du type de celui qui nous est proposé autour de la métaphore conceptuelle, notamment avec Langue française 204, Le conflit conceptuel: de la grammaire à la métaphore (Fasciolo et Neveu 2019). Les discussions présentées apportant précisément un contraste éclairant pour mettre en relief les moyens et les buts spécifiques des lexicologues-lexicographes, qui ne se comprennent que dans une perspective approfondie de linguistique appliquée, inaugurée en syntaxe française avec Maurice Gross remplaçant «théorie» par «méthodes». J'y reviens en section 2. L'opposition sens propre/sens figuré n'interviendra ici qu'en tant qu'outil de classement pour répartir les verbes dans le dictionnaire et préciser des critères justifiant les décisions de dédoublement d'entrées, une exigence prioritaire dans ce type de linguistique.

Jusqu'à quel point les décisions sont-elles indifférentes au choix du support, papier et/ ou écran, quand elles sont envisagées au niveau d'analyse le plus général, sachant qu'un dictionnaire a deux ancrages? Un ancrage lexicologique : sont en jeu des théories qu'on peut historiquement dater. Un ancrage technologique (Auroux 1995, ouvr. cit.) : les auteurs de dictionnaires sont des praticiens, confrontés dans notre actualité aux ressources lexicales sur support numérique, dont Maurice Gross, rappelons-le, aura été l'initiateur en syntaxe pour le français de la deuxième moitié du $20^{\text {ème }}$ siècle. Remarquons qu'au $21^{\text {ème }}$ siècle, les avancées des recherches et des résultats en informatique linguistique, notamment autour de l'essor des travaux sur les collocations, font précisément débat et donnent lieu à des prises de position contrastées, plaçant l'héritage de LVF au cœur des discussions (Silberztein 2019a : 7-14). Rappelons à ce propos un positionnement polémique daté mais révélateur d'Igor 
Mel'čuk (et al. 1995 : 239) déclarant : «Un DEC simplement sur papier, c'est en quelque sorte du gâchis ", que l'on pourrait résumer ainsi. Qui travaille pour qui ?

\section{Un choix exemplaire. La classe $\mathrm{C}$ des verbes de communication et le verbe bégayer}

9 En illustration d'emplois métaphoriques de verbe, je partirai ici du verbe bégayer qui retient immédiatement l'attention puisqu'il apparaît à trois reprises dans l'illustration phrastique de LVF, notamment, en vedette, dans la présentation initiale des emplois figurés (LVF 1997 : IV). A ce titre, comme je viens de l'indiquer à propos de la notion de phrase simple, un dictionnaire est proche, dans ses visées d'utilisation, des manuels d'apprentissage de la grammaire, dont Jean Dubois et Françoise Dubois-Charlier furent également des rédacteurs dans les débuts de leurs productions d'ouvrages. On ne s'étonnera pas de cette proximité dictionnaires/manuels, si on considère qu'il s'agit de deux activités qui relèvent de la linguistique appliquée, dont le lieu d'articulation dans nos sociétés est la didactique de l'écrit.

Pour bégayer, les phrases d'exemples qui sont données à l'appui de la classification sont les suivantes, le tilde servant à abréger l'illustration limitée à 60 caractères ${ }^{8}$ :

(1) L'histoire bégaie (LVF 1997, p. IV)

(2) On b depuis l'enfance (LVF 1997, classe C1a, p. 4)

(3) L'histoire b de temps à autre (LVF 1997, classe C4a, p. 32)

(4) On b à Paul une excuse, qu'on n'a rien fait (LVF, classe C2a, p. 17)

11 Rapprochons d'abord la phrase élémentaire ou noyau de présentation (1) de la phrase (3) qui en est une redite élargie en phrase simple. Le circonstant facultatif de temps à autre sert ici à faciliter l'interprétation temporelle/aspectuelle du verbe conjugué au présent de l'indicatif, en l'absence de tout contexte. Il est mis à contribution en vue de réduire les ambiguïtés. Les phrases simples dans les dictionnaires sont des énoncés brefs enrichis d'ajouts utiles au calcul du sens.

Le verbe bégayer n'a pas d'homonyme susceptible de conduire à un dédoublement d'entrées. A ce titre il n'appartient qu'à l'hyperclasse des verbes $C$ de communication. Par contraste, considérons un verbe comme voler dans la classification et les phrases simples suivantes :

(5) Les oiseaux $*$ dans le ciel (LVF 1997, classe M1a, p. 180)

(6) On a P sur la facture, sur la nourriture, sur le poids (LVF 1997, classe N1b, p. 206)

Celui-ci est répertorié dans plusieurs hyperclasses. Parmi elles, en sens propre, la classe $\mathrm{M}$ des verbes de mouvement et la classe $\mathrm{N}$ de type «munir/démunir ». Les phrases (2) et (4), quant à elles, correspondent à des sens propres avec, comme définitions générales du sens de bégayer, respectivement « exprimer par cri, parole, son » dans la classe $\mathrm{C} 1$ et "dire ou demander qc » dans la classe C2. Conformément aux choix méthodologiques du dictionnaire, cette différentiation sémantique se double, à travers la notion de schème, d'une caractérisation syntaxique systématique qui départage le verbe selon qu'il est intransitif ou transitif. Dans ces conditions, l'emploi figuré examiné ici avec (1)-(3), est cadré syntaxiquement dans une construction intransitive du verbe, ce qui exclut le verbe dans son emploi transitif (4). La combinaison du verbe et du nom, avec l'histoire bégaie, fait alors jouer un mécanisme d'apparentement par transfert ou métaphore, conçu, on le sait, comme allant du propre au figuré. On y matérialise à travers un rapport pédagogique traditionnel français/latin/grec de 
grammaire et philologie, l'idée même de sens comme trajet d'interprétation. Ce type de relation entre mots et phrases est d'autant plus naturel ici que le terme technique de «métaphore » réunit avec la " métonymie " ${ }^{9}$ les deux figures que nous intégrons depuis Dumarsais et Fontanier ${ }^{10}$ à la grammaire courante ordinaire, notamment scolaire.

Le verbe bégayer présente un intérêt bien précis pour les auteurs de LVF d'où sa place dès l'introduction à l'ouvrage, en page IV à propos des structures syntaxiques. Observée en emploi intransitif, la métaphore s'y explique par une restriction de sélection sur un groupe nominal, unique, le sujet. Le facteur déclencheur du sens figuré en est d'autant plus facile à localiser. On fait alors appel à des oppositions binaires humain/ non animé et concret/abstrait qui traversent les modèles théoriques et/ou appliqués notamment avec le célèbre exemple de Noam Chomsky, directement transposable en français, également construit sur un verbe intransitif avec sujet abstrait :

(7) Colorless green ideas sleep furiously (Syntactic Structures, 1957, p. 15)

(8) D'incolores idées vertes dorment furieusement

15 Il est une fois de plus commenté dans Fasciolo et Neveu (2019, ouvr. cité : 13-14) et situé comme relevant de la métaphore comme conflit conceptuel. L'exemple de Chomsky m'a été cité par Jean Dubois et commenté explicitement (Balibar-Mrabti, 2002 art. cit. : 97-99) quand il m'avait fourni avec l'aide de Françoise Dubois-Charlier des petits programmes de tris croisés pour extraire des cas d'emplois métaphoriques de verbes par une consultation "active» de son dictionnaire. Examinons la position de Jean Dubois rédacteur de LVF. Elle arrive en conclusion de sa conception de la métaphore dans le dictionnaire. En voici un large extrait :

On peut produire n'importe quelle phrase métaphorique à partir des sens " concrets » par la simple substitution d'un $\mathrm{N}$ abstrait à un $\mathrm{N}$ concret ou à un $\mathrm{N}$ humain* en un point quelconque de la construction basique indiquée en général par le sens [01] (des idées saugrenues glapissent dans les textes philosophiques de Paul), possible à partir des constructions à métaphorisation du sujet [le renard glapit, l'enfant glapit], phrase " abstraite », que l'on peut rendre plus "littéraire » avec la nominalisation (le glapissement des idées saugrenues dans ses textes témoigne de l'aigreur de l'auteur), produisant ainsi des phrases proches des discours de paraphrènes, qui ont d'ailleurs été prises pour des textes "poétiques", ou des phrases dites asémantiques, parfaitement interprétables si l'on se place dans une optique décalée, et d'autant plus "profondes» qu'elles exigent des commentaires plus circonstanciés, susceptibles de plusieurs « lectures », comme la célèbre phrase de Chomsky sur les « idées vertes ». * C'est moi qui souligne.

Dans ce jugement qui n'a rien d'original en soi, je discerne un positionnement, évident pour les linguistes de la génération de Jean Dubois formés dans l'université française, se démarquant des approches purement littéraires de leur génération (années 20) et affectionnant un rôle d'observateurs distanciés vis-à-vis de leurs contemporains en critique littéraire. A ceci près, et la nuance est de taille, qu'ils en sont par ailleurs des connaisseurs avertis, à travers un cursus de formation en grammaire et philologie largement centré sur la littérature comme lieu d'observation des textes en langues dites « mortes ».

Tout d'abord une remarque dans l'interview de Jean-Claude Chevalier, historien de la syntaxe et vieux compagnon de route de Dubois, assisté par le sociolinguiste Pierre Encrevé (2006, ouvr. cit.). « Dans la conversation avec Dubois, les dictionnaires tiennent toujours une grande place» (p. 159). Le centre d'intérêt permanent de Jean Dubois linguiste aura été, à l'évidence, de mener à bien une modernisation de la lexicographie. J'ajoute ici une indication précieuse que nous apporte la note écrite, que Dubois a 
envoyée en complément de ses réponses orales (p. 164-166), portant sur «formes » et " formalisme », et qui furent jugées difficile à interpréter. A ses yeux (p. 164), sur cette question complexe dont il cherche à faire le bilan critique avec le recul des années écoulées, de Martinet à Maurice Gross, il n'y avait " pas de rupture avec les philologues et grammairiens". J'interprète cette remarque en rapport avec son travail de lexicographe-lexicologue dont on retrouvera l'écho dans le dernier article de Chevalier (2010) déjà cité, "César Chesneau Dumarsais et Maurice Gross. Deux révolutions épistémologiques en miroir ».

Une chose est sûre, la phrase minimale l'histoire bégaie suscite l'adhésion mais elle s'accepte avec l'intuition qu'elle n'est pas tout à fait ordinaire. C'est cette restriction qui joue initialement à l'appui de son traitement à l'intérieur d'une sous-classe d'emplois, désignés comme étant figurés, conformément aux catégorisations des dictionnaires usuels pris comme sources de l'usage, dont Jean Dubois a fait explicitement état quand il signale son report systématique à la série historique des Larousse (Balibar-Mrabti 2002, art. cit. ; Dubois et Dubois-Charlier 2010). Bien entendu, tout lexicographe exigeant ajoutera des investigations sur corpus, en plein essor avec la documentation automatique. J'y reviens en section 3.

Reportons-nous en page IX à la présentation de la nature des sujets, où sont énumérés leurs critères généraux avec leurs notations abrégées : sujet humain noté (qn), animal (an), chose ( $q$ c), etc. On remarque que les substantifs dits «abstraits » n'en font pas partie. Pour mieux comprendre cette omission il faut aller à la «Rubrique Phrase » en page VIII et aux indications déjà données à propos des choix de substantifs sujets de verbes. En dehors de on (dont le choix est en continuité avec le premier Dictionnaire de l'Académie) LVF recherche un terme générique du domaine pragmatique et donne, pour exemples, des sujets humains comme le céramiste, le chirurgien. Ces choix se justifient quand on adopte le point de vue des sens propres. Ils perdent de leur clarté pour caractériser les sens figurés. Recourir à la notion de nom abstrait est la solution proposée à défaut des autres solutions : une solution, déjà inscrite dans la tradition, à valeur programmatique ${ }^{11}$.

La sous-classe $\mathrm{C} 4 \mathrm{a}$ est limitée à 10 entrées. Parmi elles, 4 verbes où prend place bégayer que complètent balbutier, gronder, mentir. Tous étant du type "dire, parler ", avec sujet non-animé. Je relève une précision. Devançant une observation qui saute aux yeux, le très faible volume de $\mathrm{C} 4 \mathrm{a}$ (qui n'est pas une exception dans le dictionnaire mais frappe en contraste avec d'autres sous-classes très volumineuses) les auteurs nous précisent ici que « les images et métaphores n'ont pas été répertoriées systématiquement, leur syntaxe restant celle des verbes de communication au sens propre : la rivière chante, le vent chante dans les branches ». Donnons leur appui en phrases simples :

(9) Le projet, la science balbutie encore

(10) La colère gronde dans les usines

(11) Les indices, les documents ne mentent pas

21 Le couplage proposé entre $\mathrm{C} 1 \mathrm{a}$ et $\mathrm{C} 4 \mathrm{a}$ emprunte à une répartition usuelle de notre héritage en lexicographie papier du $20^{\text {ème }}$ siècle indiqué plus haut qui attribue à la sélection d'un nom abstrait le déclenchement d'une intuition de sens figuré pour des verbes comportant tous un sens propre avec un sujet humain (balbutier, mentir), animal et/ou humain (gronder), dont l'énumération a été signalée en page IX. 

jusqu'au Nouveau Larousse Illustré1 ${ }^{12}$, on retrouve notre type d'emploi figuré avec sujet non-animé abstrait illustré par la phrase suivante attribuée à Victor Hugo ${ }^{13}$ :

(12) Au XVI ${ }^{e}$ siècle, la France littéraire BEGAYAIT encore

Cet emploi dit figuré de bégayer emporte là encore facilement l'adhésion. En est-il pour autant à considérer comme pleinement ordinaire ? Ce qui frappe ici, c'est qu'il existe dans la présentation de LVF un deuxième exemple d'emploi figuré avec le verbe éponger qui est d'emblée beaucoup moins embarrassant à juger quand il est exemplifié avec éponger un déficit budgétaire. Ici comme pour bégayer le déclencheur de la figure est comme l'histoire également catégorisé comme étant un nom non-animé abstrait. De fait les noms abstraits en position de complément de verbes ont fait l'objet de nombreuses analyses au LADL et au Laboratoire de Linguistique Informatique (LLI) de Gaston Gross.

Dans le cadrage théorique des verbes supports, comme noms prédicatifs, les substantifs abstraits sont rattachés aux sens propres. Même démarche dans les listages de verbes supports dits « appropriés ». Est-il utile de noter dans ces classements syntaxiques qu'il existe des sens figurés? Un constat. LVF ignore dans sa terminologie les verbes supports ${ }^{14}$ et privilégie, on l'a vu, le terme d'opérateur emprunté à Harris. De leur côté, le LADL et le LLI ont réduit la catégorie de "sens figuré » à des remarques occasionnelles, externes aux classifications proposées ${ }^{15}$. Gaston Gross $(1996$; 2012, ouvr. cit.) prend en compte les indications de registres de la tradition lexicographique et signale des tournures de "langue soutenue " à propos des locutions ${ }^{16}$, ce que fait d'ailleurs LVF en présentation générale, en termes de niveau de langue, ici « littéraire " (p. XIV). On relèvera chez Maurice Gross un point de vue ordinaire partagé du même ordre, quand je lui avais demandé de présenter ses premiers automates de grammaires locales dans Grammaire des sentiments (Balibar-Mrabti 1995a). Il avait choisi de désigner comme des « formes stylistiquement enrichies » des tournures imagées (M. Gross1995, section 5, p. 78-79). Parmi les exemples mis en discussion, entraient en jeu des phrases comme Un éclair de rage traversa le regard de Luc ou La nouvelle mit une expression de joie dans le regard de Luc, qui sont en continuité dans le même numéro avec mon inventaire "Une étude de la combinatoire des noms de sentiment dans une grammaire locale " (Balibar-Mrabti 1995b : 88-97), abordée identiquement à partir d'une description des degrés de figement à considérer comme relevant du français contemporain ordinaire. Ce genre d'exemples, sans signature d'auteurs et dont l'acceptabilité hors de tout contexte est intuitivement variée, appelle de sa part la note 12 (p. 78) suivante : « On remarquera que le passé simple, d'emploi limité aujourd'hui, est naturel dans ces clichés [c'est moi qui souligne] ». Je reviens sur la fonction du cliché en section 3.

Je m'intéresserai maintenant à la légitimation de la phrase (1) comme phrase non signée, rattachée à (12) qui est une phrase d'écrivain, dans la ligne des phrases d'auteurs dont le dictionnaire Littré est emblématique au $19^{\text {ème }}$ siècle. Le fait de placer dans LVF l'emploi métaphorique de bégayer sur pied d'égalité avec les sens propres l'intègre aux exemples normés que le dictionnaire a pour but de classer. D'où la question suivante à laquelle il faut répondre. Qu'est-ce qu'une métaphore normée dans un dictionnaire? Pour s'en faire une idée un tant soit peu précise, il faut s'interroger sur les tenants et aboutissants d'une notion clé en lexicographie : la phrase forgée de dictionnaire que les rédacteurs des dictionnaires Robert ont beaucoup analysée, notamment Alise Lehmann (1995a). La phrase forgée comme la phrase simple n'est pas définie clairement d'un point de vue syntaxique. A ce titre il est préférable de partir, 
pour la caractériser, de la notion d'énoncé ou de fragment de texte quand elle résulte directement d'une extraction à partir d'un cotexte, ce qui est le sens même de la notion d'exemple : « ce qui extrait » (Rey 1995 ; Fournier 2007). Le qualificatif «forgé » signale, comme son nom l'indique, une intervention, ou refonte, du lexicographe afin de proposer les « bons exemples » de phrases, aptes à vérifier un sens assorti de propriétés lexicales et de propriétés syntaxiques. Cherchons ici des sources pour L'histoire bégaie en français actuel.

\section{Le cliché comme donnée d'observation préthéorique au $20^{\text {ème }}$ siècle}

\subsection{Les exercices d'acquisition en grammaire et style : le manuel d'exercices de Charles Bally}

Dans la constitution de son vaste corpus de phrases à l'échelle d'un dictionnaire traditionnel Jean Dubois poursuit pour LVF des habitudes lexicographiques de compilation sur corpus qui traversent les époques, du papier au numérique. Notamment, il accumule des fiches pour établir ses entrées verbales ${ }^{17}$. Elles sont un moyen d'asseoir le «bon usage $~^{18}$ en grammaire, dans une conjoncture donnée, ici à l'aube du $21^{\text {ième }}$ siècle. Compilations et phraséologies ${ }^{19}$ vont de pair. Englobées dans l'exigence d'attester les données, celles-ci permettent d'élargir les classifications en direction des locutions et de leurs variantes intermédiaires, du libre au figé. Elles vont également de pair avec un héritage de listages de synonymes. Ce type de démarche hérite des méthodes pédagogiques en grammaire et style. Je dirai même que LVF se place de façon intéressante ici dans la continuité des travaux de Charles Bally, philologue, linguiste mais aussi pédagogue, à qui la montée en puissance de l'étude contemporaine des collocations a redonné une modernité.

Attardons-nous un instant sur les activités complémentaires de Charles Bally pédagogue. Il n'est pas inintéressant de remarquer qu'il a placé en annexe de son Traité de stylistique française ([1909] 1951) un manuel d'exercices (Volume II, 1951, 3 ème édit.), en continuum avec les ouvrages scolaires de son époque, dans lesquels on s'exerçait à l'acquisition des sens figurés, mêlant en vrac des exemples anonymes, signés, tirés de textes et/ou d'observations au coup par coup dans la conversation. Citons dans son manuel l'exemple suivant (p. 153), recyclé par Flaubert écrivant Madame Bovary et spécialiste de la traque des «idées reçues »:

Le char de l'état navigue sur un volcan

Cette phrase, jugée ridicule au $19^{\text {ème }}$ siècle par les grands écrivains, permet le repérage scolaire d'un cliché, le char de l'état, le télescopage des images qu'elle présente en facilitant l'analyse critique. On remarque qu'elle est présentée dans le manuel sans signature d'auteur. Le jeu présence/absence des signatures d'auteur est ici typique de visées pédagogiques qui sont en continuum avec la fabrication des exemples de sens figurés par les lexicographes. J'y reviens en section 3.2. Allons plus loin. Il contribue à façonner un regard, ni péjoratif ni admiratif, donc neutre et objectivant, sur nos données d'observation partagées.

Cette compilation des usages qui prend la forme d'une "grammaire et style en exercices» est un prérequis, d'ordre socioculturel, qui précède, à ce titre, la 
proposition du modèle théorique qu'a cherché à donner, en stylistique, dans une perspective purement synchronique, l'éditeur de Saussure, adversaire des recours à l'étymologie jugée dépassée pour délimiter "les faits d'expression $»^{20}$. Or l'analyse approfondie de la stylistique, chez Bally théoricien, porte centralement sur le statut de la littérature qu'il souhaite placer dans un continuum avec la description linguistique. Cette position est revisitée en détail par Anna Maria Curea (2015) qui en démontre l'actualité. Insistons sur l'enjeu que représentent les clichés ${ }^{21}$ dans ce débat, ancré en durée longue, sur la fonction du fait d'expression "littéraire " en langue générale normée. Dans mon approche métalexicographique, mon but est ici de sérier une transmission de phrases simples auxquelles le rôle scolaire donc social des dictionnaires et des grammaires usuelles confère une valeur canonique permettant de construire nos normes ${ }^{22}$.

Regardons dans le manuel d'exercices de Bally son volumineux «tableau synoptique des termes d'identification avec leurs principaux synonymes» (p. 223-264). Parmi eux, prend place la rubrique " caractères de la parole ", détaillée sous la partie plus générale «Expression et communication de la pensée ». J'en donne ici un aperçu sans reproduire intégralement la liste de mots synonymes proposés, dont les fonctions dans le système à visée ontologique de Bally ne vont pas nous intéresser directement ici. On y trouve le classement suivant (p. 245):

a) Parler : Se taire.

b) Parler beaucoup : P. peu. [...] Jaser, bavarder, babiller ; répéter, ressasser [...]

c) Parler haut ou fort :P. bas. Elever : baisser la voix ; crier, s'écrier ; murmurer, chuchoter [...]

d) Parler bien: P. mal. Prononcer, articuler bien ou mal; parler distinctement: bégayer*, balbutier, bredouiller, grasseyer, etc. Parler couramment [...] *souligné par moi.

31 Revenons à la sous-classe syntaxique $\mathrm{C} 4 \mathrm{a}$ où apparaît (p. 32) le verbe bégayer en emploi figuré et juxtaposons le manuel de Bally avec les formules que LVF 1997 liste, en colonne 2, dans sa rubrique Opérateur :

- balbutier (abs)loq faible

- bégayer (abs)loq répété

- gronder (abs)loq menace

- mentir (abs)loq faux

On y perçoit immédiatement une continuité de savoir-faire ${ }^{23}$. L'apparentement des procédés utilisés va de pair avec des directions d'interprétation aspectuelles, intensives ou autres des verbes énumérés. De ce point de vue, LVF réalise une mise en ordre, concertée, sur des données préalables qu'apportent les travaux et les manuels antérieurs les plus exigeants ${ }^{24}$. Plutôt raffinées et agréablement lisibles pour un utilisateur cultivé du $20^{\text {ème }}$ siècle, même non initié, ce type de données fournit, on le voit, des éléments de traitement sémantique, stabilisés dans la durée. Mais ces directions déçoivent sous l'angle de la syntaxe donc de la construction des phrases et c'est là qu'intervient l'apport original de LVF qu'étoffera le futur Dictionnaire électronique des mots (DEM) : proposer leur « réécriture » sous forme d'unités disposées en lecture gauche droite sur l'axe syntagmatique, avec en arrière-plan de cette invention, les schèmes de construction syntaxique qui sont l'ossature de la classification. Appelons formules d'opérateurs ces combinaisons originales d'étiquettes syntactico-sémantiques. 

sites. Notamment à partir d'un usage périodiquement renouvelé à travers le discours politique des journalistes d'opinion. Cet usage passe par une attribution plus ou moins approximative à Marx qui est la suivante: L'histoire ne se répète pas. Elle bégaie. Il est indissociable de certaines variations de formulation, incluant le cas échéant des traductions qui ne lui enlèvent pas son sens général. Sa présence multiple inclut naturellement ici la possibilité d'aboutir à une phrase forgée ordinaire de dictionnaire comme L'histoire bégaie avec (1), où se trouve supprimée la première phrase d'introduction, ce qui entraine la réintroduction du sujet l'histoire en remplacement de l'anaphorique elle, comme on éliminerait ailleurs les déictiques. Pour améliorer l'interprétation on ajoutera, on l'a vu, dans LVF avec (3), le circonstant de temps à autre.

Une remarque s'impose. La source en français numérisé renvoie essentiellement à des contenus journalistiques ${ }^{29}$. C'est un fait marquant de notre modernité que de tendre à 
remplacer les textes littéraires, comme garants du bon usage, par la presse écrite en pleine ascension à travers une notion de plus en plus établie, la littératie ou littéracie (par traduction de literacy), ce qui permet d'englober tout type d'écrit sans valorisation particulière de la littérature des écrivains. Soulignons, de ce point de vue, la place des journalistes dans la reconduction des pratiques accrochées historiquement à l'enseignement de rudiments rhétoriques, notamment l'usage des métaphores, dès lors qu'ils seront considérés comme opératoires pour convaincre, dans une optique classique de persuasion. Un objectif survalorisé en ce début de $21^{\text {ème }}$ siècle ${ }^{30}$. Ici l'attribution de la parole humaine à une entité abstraite comme l'histoire, ou la France, procède d'un arsenal de moyens langagiers inscrits dans la longue durée scolaire des arts d'écrire et de penser qui enseignent la personnification, l'allégorie, la prosopopée. On y remarque différentes variantes de formulation: temps du verbe, ajouts de circonstants ainsi que de mini-contextes, dont la phrase simple de dictionnaire s'affranchit parce qu'elle recherche par divers procédés empiriques incluant des choix de ponctuation un énoncé minimal à visée canonique : la phrase simple ${ }^{31}$. Dans cette recherche, on remarque également que les signatures d'auteur ne sont plus la priorité.

Alise Lehmann (1995a, art. cit.) a détaillé ces manipulations dans le passage concerté du Grand Robert au Petit Robert. Elles expliquent sans aucune différence de méthode les confections d'exemples dans LVF, avec comme exigence matérielle initiale chez les lexicographes : le gain de place. Les dictionnaires papier ont un volume contraint qui, on le sait, est périmé sur support électronique quand on énumère de gros volumes de phrases mais la recherche des énoncés brefs perdure ${ }^{32}$. Cette reconduction est un sujet d'analyse qui reste largement à explorer. Reprenons une des conclusions de l'auteure (p. 119): "il y a un continuum exemples signés/exemples observés non signés/exemples construits. De plus, la répartition des exemples signés en énoncés littéraires / non littéraires renvoie à la question de la classification des sources, qui devrait être repensée ». Venant à l'appui de cette conclusion, nos attestations sur corpus conduisent à s'interroger sur le poids de la Presse contemporaine ${ }^{33}$. L'intérêt, de plus en plus grand pour les échanges dans les réseaux sociaux, qui donne priorité aux usages dits " permissifs »- quand il est accroché au choc contemporain de l'oral avec un écrit éloigné des formes dites standard - est loin d'en épuiser les aspects les plus importants pour un historien de la syntaxe. Une des propriétés observées ici est le réajustement des temps. De fait LVF retouche les temps et privilégie le présent et le passé composé de l'indicatif comme M. Gross (1975, ouvr. cit.) dans la ligne des recommandations «modernistes" du Français fondamental (Gougenheim [1956] 1966, ouvr. cit.).

\section{Eléments pour une conclusion. Coder un calcul du sens à la frontière des traitements automatiques}

Les auteurs de LVF sont délibérément elliptiques quant à leurs a priori de méthodes. De quelle théorie grammaticale est-il question exactement? On sait que Jean Dubois et Françoise Dubois-Chalier se sont affranchis de toute référence directe à une école linguistique précise et parlent de méthodes de la grammaire transformationnelle et distributionnelle qu'ils qualifient de " classiques » (LVF 1997 : III). Prenant leur distance avec la grammaire générative à propos des dictionnaires à réaliser et proches sur ce point des positions du LADL, les auteurs adoptent ce qualificatif habile qui leur permet 
d'aménager leur terminologie placée à la rencontre de la grammaire scolaire et des méthodes de la linguistique structurale américaine dont Jean Dubois recommandait la lecture à ses étudiants dans les années 1960 à travers Henry A. Gleason ${ }^{34}$, qu'il complétait par des renvois directs à Zellig Harris et Noam Chomsky élaborant leurs premiers modèles transformationnels ${ }^{35}$. Ces appuis initiaux sont significatifs de la conception de la syntaxe que Jean Dubois souhaitait mettre en œuvre dès la fin des années soixante ${ }^{36}$ pour moderniser la lexicographie française du $20^{\text {ème }}$ siècle.

De plus, le positionnement dans LVF correspond à un souci délibéré de s'éloigner des innovations terminologiques d'où une attitude de Jean Dubois fréquemment observée en didactique, faire jouer le réemploi d'une terminologie scolaire banalisée et officialisée, installée dans la durée. On retrouve cette attitude jusque dans ses classifications les plus tardives. Un exemple qui traverse son œuvre : une notion comme celle de «syntagme nominal», avec ses connotations en linguistique structurale traduisant noun phrase, qu'il rectifie selon les cas en "groupe du nom ", allant même jusqu'à remplacer "syntagme " par "syntaxe ». C'est souvent la position des bons grammairiens ${ }^{37}$, comme des lexicographes, conscients de la nécessité d'être compris par les locuteurs non spécialistes, en même temps qu'ils cherchent à faciliter la mémorisation de leurs principes d'analyse quand ils sont appréhendés à partir d'une lecture papier. L'enjeu est alors de revoir et corriger les contenus de définition du vocabulaire grammatical et sémantique utilisé, compte tenu des avancées en sciences du langage, notamment ici avec l'apparition des grammaires en chaîne appliquées au LADL par Morris Salkoff ${ }^{38}$ traducteur du français vers l'anglais.

41 De fait Jean Dubois invente, je dirai même bricole, ses propres codages alphanumériques ainsi que ses notations abrégées, émancipées des formalismes logicomathématiques et distanciées des calculs proprement informatiques. En privilégiant une analyse surfacique des phrases (sans arborescences) visant la caractérisation d'emplois prototypiques pour les verbes, il détaille méticuleusement ${ }^{39}$ ses combinatoires d'éléments discrets ${ }^{40}$. Revenons sur ses formules d'opérateurs, telles qu'elles sont listées, en colonne, pour chaque entrée verbale considérée, dans LVF 1997. Le mixte de renvois à des unités d'interprétation de sémantique et de syntaxe y est disposé linéairement. Le procédé d'exposition s'apparente à des moyens mnémotechniques de lecture et d'écriture rapides ${ }^{41}$. Il s'apparente également à la tradition des traductions du latin au français dites «juxta-linéaires » qui s'observent encore en didactique ${ }^{42}$. J'ai déjà décrit ailleurs (Balibar-Mrabti 2007) à propos de l'utilisation massive dans LVF du pronom sujet humain on, comment sont recyclées et modernisées, pour une large part, des habitudes antérieures au structuralisme en linguistique et familières à toute entreprise réussie d'élaboration d'un dictionnaire, qu'il soit électronique ou papier. Sont ciblées ici des types de rencontre entre une étape historique datée de la théorie linguistique (donnant priorité à la syntaxe) et ses applications à travers des programmes de réalisations lexicales détaillées laissées ouvertes $^{43}$, entreprises à grande échelle. Celles-ci conduisent inévitablement à des décisions à prendre au coup par coup, sans qu'on puisse parler péjorativement de solutions ad hoc ni les considérer par abus de langage comme étant "impressionnistes " en syntaxe (Balibar-Mrabti 2008). C'est d'ailleurs cet espace de recherches, nécessitant une nouvelle science du détail en grammaire, dont des spécialistes comme Jean Dubois et Françoise Dubois-Charlier avaient l'expertise, comme philologuesgrammairiens et comme lexicographes chez Larousse ${ }^{44}$. 
Notons en conclusion que dans leur dernière publication (Dubois et Dubois-Charlier 2010, art. cit., p. 33), les auteurs soulignent qu'ils ont utilisé des rubriques traditionnelles «que l'on peut trouver (moins détaillées) sous une forme ou sous une autre dans un dictionnaire de langue ou un autre ». J'en ai indiqué ici quelques pistes essentiellement sémantiques (section 3.1). Et ils reprécisent leur apport contemporain à la réalisation des dictionnaires, qui est d'ordre syntaxique. Dans leur dictionnaire électronique des mots (DEM) en cours de réalisation, remarquons la rubrique CONT, désignant ce qu'ils appellent le contexte ou le contenu. Ils nous déclarent (p. 43) : «Sur l'axe syntaxique, ce qui est écrit avec CONT forme avec le mot d'entrée une ébauche de phrase simple ou prototypique ». On relèvera le terme «ébauche » et sa prudence pour suggérer l'ampleur de la distance qui sépare de ses données observables en langues naturelles, ici le français écrit actuel, un cadre d'analyse rigoureux exprimé en « rubriques ». Les données étant constituées par des phrases effectives, sélectionnées et refaçonnées par les linguistes. On notera également que la rubrique CONT est une réponse originale des auteurs aux problèmes d'approches syntactico-sémantiques du lexique par verbes supports et « classes d'objets » (G. Gross 2012, ouvr. cit. ; Le Pesant et Mathieu-Colas 1998) dont ils furent les analystes parmi les plus proches.

A propos des métaphores, sont intervenus dans LVF des facteurs socioculturels relevant du cliché comme figure de style permettant d'emporter l'adhésion et de peser, à la marge des sens propres, en faveur d'un choix d'illustration comme L'histoire bégaie. Faudrait-il définir ici un type d'argument d'autorité incorporé aux stratégies mises en œuvre pour élaborer les dictionnaires de langue générale ? La question est d'autant plus cruciale qu'on sait qu'un dictionnaire est, toutes proportions gardées ${ }^{45}$, l'outil majeur de notre modernité (préparé par l'école) pour consulter et vérifier nos normes collectives d'utilisateurs instruits. Et il ne s'agit pas seulement d'y relever des variations hautement significatives dans l'évolution historique des formes et des sens. Il est tout aussi essentiel d'y évaluer les contreparties obligées de la variation et d'y sérier des stabilités dont la reconduction va de pair avec un ensemble d'aménagements modulables ${ }^{46}$. Ces reformulations se réalisent précisément grâce aux auteurs de dictionnaires quand ils inventent leurs phrases forgées, admirables de simplicité et de nature ${ }^{47}$ dans LVF, et contribuent à matérialiser notre imaginaire partagé de la phrase dite "libre " avec ses règles à l'écrit. A ce titre, nous héritons en langue française d'un débat initial posé au $17^{\text {ème. }}$ siècle et bien connu en métalexicographie. Depuis que les acquis scolaires et les facilités d'impression ont considérablement élargi le statut d'écrivain (même situation avec la photographie et le cinéma), posons-nous collectivement la question suivante. Entre grammaire et littérature d'auteurs, incluant la part grandissante des journalistes à travers les moyens contemporains du numérique pour tous, qui est au service de qui ?

De ce bref examen concernant les conditions d'élaboration d'une base d'observation des verbes en emploi, dont LVF est un cas remarquable par sa précision et son ampleur, il ressort les caractéristiques suivantes. Dès qu'on passe à des sens abstraits et/ou figurés les consensus mettent en jeu non seulement la norme mais également toute la complexité des usages, partiellement masquée par la notion d'intuition des sujets parlants. Ce recours à l'intuition, pour justifier les tris entre phrases grammaticales, phrases agrammaticales, phrases stylistiquement enrichies, est actuellement fragilisé par les outils informatiques que nous mettons au service de l'observation des données sur gros corpus. De fait, nous adoptons un regard renouvelé sur les textes désormais 
massivement lus et écrits en langues numérisées. Recentrer nos analyses sur la métalexicographie, envisagée en durée longue, permet d'attirer l'attention sur un mixte plus ou moins explicite de critères que la linguistique appliquée véhicule par les dictionnaires et l'enseignement de la grammaire scolaire. Les manipulations de citations d'écrivains y sont incontournables parce qu'elles prennent place dans une pratique (suggérée) d'imitation inventive dont jouent activement les journalistes actuels, notamment à travers un héritage rhétorique commun, qui contribue directement à cimenter une compétence écrite "ordinaire» des sujets parlants et "écrivants ». Ici le couplage phrase simple/phrase forgée de dictionnaire est une des clés de l'analyse.

\section{BIBLIOGRAPHIE}

Achour, C. (1985). Abécédaires en devenir. Idéologie coloniale et langue française en Algérie. Alger : Entreprise Algérienne de Presse.

Anscombre, J-C. et al. (2016). (éds) La phrase autonome : Théorie et manifestations. Berne : Peter Lang Publishing.

Anscombre, J.-C. (2005). « Les proverbes : un figement du deuxième type ? », in A. Balibar-Mrabti \& C. Vaguer (éds) Le semi-figement, pp. 17-33. LINX n53. Nanterre : Editions de l'Université Paris $\mathrm{X}$.

Auroux, S. (1995). La révolution technologique de la grammatisation, introduction à l'histoire des sciences du langage. Liège : Mardaga.

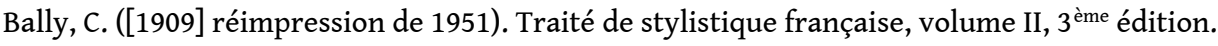
Paris : Klinksieck.

Balibar-Mrabti, A. (2017a). « Le français écrit numérisé », in La linguistique, vol. 53 (1) Varia, pp. 129-147. Paris : Presses universitaires de France.

Balibar-Mrabti, A. (2017b). Compte rendu-discussion sur l'ouvrage de J.-C. Anscombre et al. (2016) La phrase autonome. Berne : Peter Lang, in Cahiers de lexicologie, 2016 - 2, n 109, pp. 247-254. Paris : Classiques Garnier.

Balibar-Mrabti, A. (2011a). «Ellipse, figement, traductions, Tomber de Charybde en Scylla », in J.C. Anscombre \& S. Mejri. (éds). Le figement linguistique : la parole entravée, pp. 267-279. Paris : Champion.

Balibar-Mrabti, A. (2011b). « Lexicographie, grammaire et lexique : une mise en perspective historique », in Cahiers de lexicologie n99, pp. 255-263. Paris : Classiques Garnier.

Balibar-Mrabti, A. (2008). Avant-Propos « Le nombre en français : un ouvrage de consultation. Morphologie, syntaxe, traitements automatiques associés », in J. Dubois \& F. Dubois-Charlier. Le nombre en français, pp. 5-8. Fernelmont/Paris : Editions Modulaires Européennes/ L'Harmattan.

Balibar-Mrabti, A. (2007). « Phrases simples et exemplification dans Les Verbes français. Une réflexion sur on comme sujet », in Langue française n¹53, pp. 92-126. Paris : Larousse. 
Balibar-Mrabti, A. (2005). « Semi-figement et limites de la phrase figée », in LINX n53, pp. 35-54. Nanterre : Presses de l'Université Paris X.

Balibar-Mrabti, A. (2004). « Lexique-grammaire et extensions lexicales. Note sur le semifigement », in Lingvisticae Investigationes Supplementa 24, pp. 23-29. Amsterdam /Philadelphia : Benjamins.

Balibar-Mrabti, A. (2002). « Exemples lexicographiques et métaphores », in Langue française $\mathrm{n}^{\circ} 134$, pp. 90-108. Paris : Larousse.

Balibar-Mrabti, A. (1997). (éd) La synonymie, Langages nº128. Paris : Larousse.

Balibar-Mrabti, A. (1995a). (éd.) Grammaire des sentiments, Langue française $n^{\circ} 105$. Paris : Larousse.

Balibar-Mrabti, A. (1995b). « Une étude de la combinatoire des noms de sentiment dans une grammaire locale », in Langue française $n^{\circ} 105$, pp. 88-97. Paris : Larousse.

Boons, J.-P., Guillet, A., Leclère, C. (1976). La structure des phrases simples en français, 1 : Constructions intransitives. Genève : Droz.

Branca, S. (1980). «L'art d'écrire de Condillac (1775); à propos de quelques règles prescriptives : traitement des ellipses et des anaphores ", in Langue française $n^{\circ} 48$, pp. 44-56. Paris : Larousse.

Bréchet, F. et al. (2017). Le Préconstruit. Approche pluridisciplinaire, Rencontres n²92, Paris : Classiques Garnier.

Chevalier, J.-C. (2010). « César Chesneau Du Marsais et Maurice Gross. Deux révolutions épistémologiques en miroir ", in Echo des études romanes, Vol. VI, n¹-2, pp. 73-83. www.eer.cz. Chevalier, J.-C. ([1968] 2006). Réédition augmentée d'une présentation par l'auteur, Histoire de la syntaxe : Naissance de la notion de complément dans la grammaire française (1530-1750). Paris : Champion.

Chevalier, J.-C. (1998). « Lexique-grammaire et histoire de la linguistique. Un lexique-grammaire : Lesclarcissement de John Palsgrave, 1530 », in Travaux de linguistique n³7, pp. 143-154. Bruxelles/Paris : De Boeck.

Chevalier, J.-C. (1997). « La synonymie dans les manuels pédagogiques du début de la Renaissance », in Langages $n^{\circ} 128$, pp. 8-24. Paris : Larousse.

Chevalier, J.-C. (1994). «F. Brunot (1860-1937) : la fabrication d'une mémoire de la langue » in Langages 114, pp. 54-68. Paris : Larousse.

Chevalier, J.-C., Encrevé, P. (2006). Combats pour la linguistique de Martinet à Kristeva, Essai de dramaturgie épistémologique. Lyon : ENS Editions.

Chevalier, J.-C., et al. (1964). Grammaire Larousse du français contemporain. Paris : Larousse.

Chomky, N. (1957). Syntactic Structures. The Hague : Mouton.

Cougnon, L. A. (2015). Langage et sms : Une étude internationale des pratiques actuelles. Louvain : Presses Universitaires de Louvain.

Courtois, B., Silberztein, M. (1990). (éds) Dictionnaires électroniques du français, Langue Française $n^{\circ} 87$. Paris : Larousse.

Curea, A. M. (2015). Entre expression et expressivité : l'école linguistique de Genève de 1900 à 1940. L'expressivité linguistique, un objet problématique de la théorie de Charles Bally. Lyon : ENS éditions. 
Dubois, J. (1962). Le vocabulaire politique et social en France de 1869 à 1872, A travers les œuvres des écrivains, les revues et les journaux. Paris : Larousse.

Dubois, J., Dubois-Charlier, F. (2015). Dictionnaire électronique des mots. (DEM). en libre accès sur le site du laboratoire ModyCo.

Dubois, J., Dubois-Charlier, F. (2010). « La combinatoire lexico-syntaxique dans le Dictionnaire électronique des mots. Les termes du domaine de la musique à titre d'illustration ", in Langages n¹79-180, pp. 31-56. Paris : Larousse.

Dubois, J., Dubois-Charlier, F. (2008). Le nombre en français. Fernelmont/Paris : Editions Modulaires Européennes/L'Harmattan.

Dubois, J., Dubois-Charlier, F. (2004). Locutions en français. En libre accès sur le site du Laboratoire MoDyCo.

Dubois, J., Dubois-Charlier, F. (1997). Dictionnaire Larousse du français langue étrangère niveau 2. Paris : Larousse.

Dubois, J., Lagane, R. (1973). La nouvelle grammaire du français. Paris : Larousse.

Dubois, J., et al. (1966). Dictionnaire du français contemporain. Paris : Larousse.

Dubois-Charlier, F. (2015). Informations sur les rubriques du DEM en libre accès sur le site du Laboratoire MoDyCo.

Dubois-Charlier, F. (1967-1975). Introduction à la linguistique, Paris : Larousse, traduction française de H. Gleason, (1961). An introduction to descriptive linguistics, New York : Holt, Rinehart and Winston.

Fairon, C. (2004). « Une étude de corpus pour éclairer la question de l'incise en français ", in Lingvisticae Investigationes Supplementa n²4, pp.195-210. Amsterdam/Philadelphia : Benjamins.

Fairon, C., Simon, A.-C. (2018). Le Petit Bon usage de la langue française, Grammaire. Louvain-laNeuve : De Boeck.

Fasciolo, M., Neveu, F. (2019). (éds) Le conflit conceptuel : de la grammaire à la métaphore, Langue française $n^{\circ} 204$. Paris : Armand Colin.

Florea, L.-S., Fuchs, C., Mélanie-Becquet, F. (2010). Dictionnaire des verbes du français actuel : constructions, emplois, synonymes. Paris : Ophrys.

Fournier, J.-M. (2007). (éd) L'exemple dans les traditions grammaticales, Langages $n^{\circ} 166$, Paris : Larousse.

Gala, N. (2018). « La simplification des textes : une aide à l'apprentissage de la lecture », in Langue française $n^{\circ} 199$, pp. 123-131. Paris : Armand Colin.

Gala, N., Zock, M. (2013). (éds) Ressources lexicales. Contenu, construction, utilisation, évaluation. Lingvisticae Investigationes Supplementa $n^{\circ} 30$. Amsterdam /Philadelphia : Benjamins.

Genette, G. (1991). Fiction et diction. Paris : Le Seuil.

Giry-Schneider, J. (1987). Les prédicats nominaux en français. Les phrases simples à verbe support. Genève-Paris : Droz.

Goody, J. (1977). The Domestication of the Savage Mind. Cambridge : Cambridge University Press. (1979) en traduction française. La raison graphique : la domestication de la pensée sauvage. Paris : Editions de Minuit. 
Gougenheim, G., et al. ([1956] 1964). L'élaboration du français fondamental (1 ${ }^{\mathrm{er}}$ degré). Paris : Didier.

Grevisse, M., Goose, A. (1936-2016). Le Bon usage. Louvain-la-Neuve/Bruxelles : De Boeck/ Duculot.

Gross, G. (2012). Manuel d'analyse linguistique. Villeneuve d'Ascq : Presses Universitaires du Septentrion.

Gross, G. (1999). Les expressions figées en français, Noms composés et autres locutions. Paris : Ophrys, Paris.

Gross, M. (1995). « Une grammaire locale de l'expression des sentiments », in Langue française $\mathrm{n}^{\circ} 105$, pp.70-87. Paris : Larousse.

Gross, M. (1975). Méthodes en syntaxe. Paris : Hermann.

Guillet, A., Leclère, C. (1992). La structure des phrases simples en français, 2 : Les constructions transitives locatives. Genève-Paris : Droz.

Harris, Z. (1976). Notes du cours de syntaxe. Paris : Le Seuil.

Harris, Z. (1957). « Co-Occurrence and Transformation in Linguistic Structure », in Language 33:3(1), pp. 283-340. Washington : Linguistic Society of America.

Lakoff, G., Johnson, M. (1980). Metaphors We Live By. Chicago : University of Chicago Press. (1986) en traduction française, Les métaphores dans la vie quotidienne. Paris : Editions de Minuit.

Leclère, C. (2002). « Emplois verbaux, distributions, métaphores », in Langue française 134, pp. 78-89. Paris : Larousse.

Le Goffic, P. (1993). La grammaire de la phrase française. Paris : Hachette.

Lehmann, A. (1995a). « Du Grand Robert au Petit Robert : les manipulations de la citation littéraire », in Lexique n¹2/13, pp.105-124. Villeneuve d'Ascq : Presses Universitaires du Septentrion.

Lehmann, A. (1995b). (éd) L'exemple dans le dictionnaire de langue. Histoire, typologie, problématique, in Langue française $\mathrm{n}^{\circ} 106$. Paris : Larousse.

Le Pesant, D. (2020). « Place de l'œuvre de Jean Dubois et Françoise Dubois-Charlier dans l'histoire de la linguistique », dans ce numéro.

Le Pesant, D., Mathieu-Colas, M. (1998). « Introduction aux classes d'objets », in Langages n¹31, pp. 6-33. Paris : Larousse.

Lusignan, S. (2004). La langue des rois au Moyen Age. Le français en France et en Angleterre. Paris : Presses universitaires de France.

Mel'čuk, I., Clas, A., Polguère, A. (1995). Introduction à la lexicologie explicative et combinatoire. Louvain-la-Neuve : Duculot.

Mel'čuk, I., et al. (1985-1992). Dictionnaire explicatif et combinatoire du français contemporain. Recherches lexico-sémantiques. Montréal : Les Presses de l'Université de Montréal.

Nouveau Larousse illustré (1897-1904). C. Augé. (éd). Paris : Larousse.

Pellat, J.-C., Fonvielle, S. (2016). Le Grevisse de l'enseignant. Grammaire de référence. Paris : Magnard.

Philippe, G. (2002). Sujet, verbe, complément ; Le moment grammatical de la littérature française, 1890-1940. Paris : Gallimard. 
Prandi, M. (1992). Grammaire philosophique des tropes. Paris : Editions de Minuit.

Rey, A. (1995). « Du discours au discours par l'usage : pour une problématique de l'exemple », in Langue française ${ }^{\circ} 106$, pp. 95-120. Paris : Larousse.

Rey, A. (1977). Le lexique. Images et modèles. Du dictionnaire à la lexicologie. Paris : Armand Colin.

Rey-Debove, J. (1997). « La synonymie ou les échanges de signes comme fondement de la sémantique », in Langages $n^{\circ} 128$, pp. 91-104. Paris : Larousse.

Riegel, M., Pellat, J.-C., Rioul, R. (1994). Grammaire méthodique du français. Paris : Presses universitaires de France.

Robert, dictionnaires, se reporter à Lehmann (1995a).

Robert \& Collins Français/Anglais et Anglais/Français, 2001, version papier. Paris : Editions Le Robert.

Salkoff, M. (1999). A French-English Grammar. A Contrastive Grammar on Translational Principles. Lingvisticae Investigationes Supplementa 22. Amsterdam/Philadelphia: Benjamins.

Salkoff, M. (1983). « Bees are swarming in the garden: A Systematic Study of Productivity», in Language, 59.2, pp. 288-346. Washington : Linguistic Society of America.

Salkoff, M. (1979). Analyse syntaxique du français : grammaire en chaîne, Lingvisticae Investigationes Supplementa 2. Amsterdam : Benjamins.

Silberztein, M. (2019a). « Les outils informatiques au service des linguistes : présentation », in Langue française $n^{\circ} 203$. Paris : Armand Colin.

Silberztein, M. (2019b). (éd) Les outils informatiques au service des linguistes, in Langue française $\mathrm{n}^{\circ} 203$. Paris : Armand Colin.

Trésor de la Langue Française informatisé, TLFi, en ligne, http://atilf.atilf.fr

Tremblay, O., Polguère, A. (2014). « Une ontologie linguistique au service de la didactique du lexique », Congrès Mondial de Linguistique Française, hal.archives-ouvertes.fr

\section{NOTES}

1. Le dictionnaire Les Verbes français comporte plusieurs versions successives. Je me réfère ici à la version papier datée de 1997 que les auteurs ont réalisée chez Larousse à l'intention des chercheurs.

2. On s'y adosse à la terminologie officielle, périodiquement actualisée, qui la donne parmi les termes à employer dans les programmes d'enseignement du français.

3. On notera qu'elle n'existe pas dans un ouvrage universitaire à visée purement scientifique comme la Grammaire de la phrase française de Pierre Le Goffic (1993), ce qui confirme sa nature scolaire et littéraire par les textes. A destination d'un public analogue d'utilisateurs, Gaston Gross (2012:17) y fait une allusion rapide et indique son ancrage, comme donnée observable, dans les textes.

4. Pour un bilan d'actualité sur cet implicite partagé je renvoie à La phrase autonome (Anscombre et al. 2016) et à mon compte rendu/discussion de présentation (Balibar-Mrabti 2017b).

5. Comme le signale explicitement dans son choix d'intitulé l'ouvrage en deux tomes Structure des phrases simples en français (Boons, Guillet et Leclère 1976; Guillet et Leclère 1992). Ou encore 
Jacqueline Giry-Schneider (1987) dans son ouvrage sur les prédicats nominaux sous-titré Les phrases simples à verbe support.

6. L'ouvrage Ressources lexicales (Gala et Zock 2013), paru du vivant des auteurs de LVF, effectue un tour d'horizon instructif sur ce point.

7. De ce point de vue, Dubois, comme Mel'čuk, a étudié avec attention dans M. Gross (1975) les commentaires de table du chapitre IV (p.160-214) qui comportent de nombreuses remarques sur les sens observés. Elles deviennent intéressantes à reprendre a posteriori quand on dispose désormais de classifications très élaborées incorporant syntaxe et sémantique. Le commentaire de la table 4 débute ainsi: "Les verbes de cette table sont sémantiquement homogènes. La grande majorité d'entre eux correspond à un sentiment " déclenché " par $N_{1}$ et éprouvé par $N_{0}$ [...] avec la forme adjectivale associée $N_{0}$ est $V$-a pour $N_{1}$ ». On peut supposer que cette table aura suggéré à Jean Dubois des potentialités classificatoires pour LVF quand il choisit d'interpréter sémantiquement ses propriétés syntaxiques de classement par schèmes et retient sa notion d'opérateur. L'ampleur des résultats obtenus, une cinquantaine de pages (LVF 1997 : 245-273) pour énumérer les entrées, avec la classe $\mathrm{P}$ des verbes "psychologiques", est une réussite spectaculaire, parmi d'autres, de la mise en place de critères syntactico-sémantiques.

8. Une précision donnée en page IX à propos de la version électronique initiale du dictionnaire, dont LVF 1997 est, rappelons-le, un type de tirage papier. Remarquons à ce propos une similitude d'ordre général avec les tables syntaxiques du LADL, notamment la publication fondatrice de $\mathrm{M}$. Gross (1975, ouvr. cit.) où la présentation, sur une seule ligne, des profils de + et de - des verbes classés obéissait à une contrainte de longueur adaptée dès son époque à la largeur des écrans. On sait que les dictionnaires ont des «mises en page " particulièrement élaborées. Sur ce point, la période historique actuelle est un cas type, dont les tendances sont encore largement à analyser, dans une conjoncture que caractérise un nouveau statut du support papier : demeuré essentiel mais désormais couplé avec les nouvelles fonctionnalités, invasives et incontournables, des langues numérisées sur écran. On l'a indiqué (section 1), c'est toute la question des correspondances entre métalexicographie et dictionnairique, devenues cruciales, en théorie comme en pratique, à propos de la place de l'informatique en linguistique.

9. La métonymie tient une place centrale dans les travaux du LADL avec la transformation dite de « restructuration" du groupe nominal rappelée par Leclère (2002 art. cit.). Pour une mise en perspective historique en durée longue, voir Chevalier (2010). Un exemple (Balibar-Mrabti (2011a), le nom propre Charybde qui a pour source les dangers de Charybde dans la locution tomber de Charybde en Scylla.

10. Remaniant Dumarsais. Pour un examen approfondi en "grammaire philosophique des tropes » je renvoie à Prandi (1992 : 106 sv.). Pour une optique métalexicographique appliquée aux classifications de Jean Dubois, se reporter à Chevalier (2010, art. cit.). Pour son traitement dans le Lexique-Grammaire, voir Leclère (2002, art. cit.).

11. G. Gross (2012, ouvr. cit.) réaborde la question en fin d'ouvrage et en souligne les difficultés d'utilisation rationnelle. Riegel (et al.,1994, ouvr. cit. :171) voit lui aussi tout le flou que comporte une telle notion dont il souligne un côté fourre-tout. Il énumère des questions traitées avec les Vsup du LADL mais il fait l'impasse sur son rôle classique dans le traitement des figures.

12. Explicitement indiqué, on l'a dit, par les auteurs en 2010 et reconnu comme source dans des échanges directs à propos des locutions brûler ses vaisseaux ou glaner des idées commentées dans mes publications.

13. Le Trésor de la Langue Française informatisé (TLFi) nous en donne la référence exacte, réécrite et abrégée dans le Larousse : "A cette époque, la France militaire parlait haut en Europe, mais la France littéraire bégayait encore » Hugo, Le Rhin, 1842, p. 422. J’y reviens en section 3.2.

14. Le Pesant (2020, dans ce $\left.n^{\circ}\right)$ fait le même constat. J'y reviens en section 4 . Chronologiquement la terminologie a d'ailleurs fluctué au LADL, passant par une notion de verbes dits " auxiliaires ", distingués des verbes dits «opérateurs ». Par leur côté novateur, les Vsup ne pouvaient que faire 
une entrée discrète dans les manuels, à visée scolaire, les plus fouillés : Riegel et al. (1994 : 232-233) et dans Fairon et Simon (2018) qui les introduit à deux reprises, p. 328 et 360.

15. A l'exception notoire de Morris Salkoff (1983) publiant dans Language, Volume 59, $\mathrm{N}^{\circ} 2$ : 288-346, «Bees are swarming in the garden: A Systematic Study of Productivity», un article mal reçu au départ puis salué par David Dowty l'indiquant comme données cruciales pour établir ses protorôles thématiques (Conférence plénière, colloque OBJETS, Relations grammaticales et rôles sémantiques, Université de Gand, Béatrice Lamiroy et Dominique Willems, organisatrices, 1997). A noter que cette étude part d'une analyse effectuée sur le français par Boons, Guillet, Leclère (1976, ouvr. cit.).

16. Notamment avec des équivalences comme écouter d'une oreille attentive = écouter d'une (façon +manière) attentive = écouter attentivement (Balibar-Mrabti 2004) qui mettent en parallèle les Ns façon, manière avec le $\mathrm{N}$ oreille comme Napp, à rapprocher ici d'un Vsup approprié, dans une analyse des groupes nominaux adverbiaux adaptée de Harris (1976). Dans cette synonymie syntaxique, je soulignais (p. 25) que la combinaison du nom oreille avec l'adjectif attentif « fait cliché dans une interprétation psychologique abstraite » qui en assure l'acceptabilité.

17. Communication personnelle à propos de la locution brûler ses vaisseaux (Balibar-Marbti 2005) mise sur fiche mais non cataloguée dans locutions en français (Dubois et Dubois-Charlier 2004, en édit. électr. sur le site de MoDyCo). Notons que LVF (1997: XI) présente son dictionnaire électronique comme un ensemble de 25610 fiches. Le terme semble bien daté. Cependant il ne faudrait pas sous-estimer ce à quoi il nous renvoie dans les méthodes de travail du passé. Par exemple, quand les lexicographes de la génération de Jean Dubois, pratiquaient des monographies lexicologiques incontournables pour entrer dans les détails du lexique (Rey 1977, ch. X : 225-237). Ajoutons pour l'anecdote un commentaire de Maurice Gross au LADL dans les années 1970 : ce que nous faisons ici pourrait se faire sur fiches perforées.

18. La notion d'usage et ses variantes de formulation est incontournable pour parvenir à une vision claire des normes $d u$ français écrit. Elle ne sera pas approfondie ici. Le débat est considérable. Il concerne précisément les choix des lexicographes de proposer ou non des exemples signés ou non signés. Même problématique en grammaire usuelle. On sait que la première grammaire Grevisse met en avant les phrases d'auteurs à l'appui de l'usage. Dans sa refonte de 2018, Cédrick Fairon maintient cette ligne. Cette option donne à réfléchir, venant d'un linguiste qui a soutenu sa thèse de doctorat sous la direction de Maurice Gross sur le traitement automatique de l'incise d'une part (Fairon 2004), et lancé, d'autre part, les premiers travaux d'analyse des sms en contexte francophone. Voir sur ce point Cougnon (2015).

19. Solidement ancrées historiquement en grammaire francophone dans les méthodes d'apprentissage par traduction (version et surtout thème) des langues sous forme écrite avec le latin et le grec ancien comme base d'apprentissage. D'où cette remarque en 2007 de Jean Dubois au cours d'un échange personnel sur la permissivité en orthographe contemporaine : le français écrit, c'est du latin.

20. «On observera une fois pour toute que les exercices [se rapportant au Traité] sont destinés uniquement à combattre l'instinct étymologique. » (Bally, [1909] 1951, volume II, ouvr. cit. p. 1).

21. Les termes de cliché ou de stéréotype sont remplacés par le "préconstruit» dans notre modernité immédiate de chercheurs, notamment par Bréchet (et al., 2017). On sait que Bally théoricien avait choisi de recycler le vieux terme de "phraséologie " dont il était proche à travers la pédagogie ordinaire de son temps (voir plus haut ma note 19).

22. Ce faisant, je ne m'aligne pas sur des problématiques du type de celles lancées par George Lakoff et Marc Johnson (1980), Metaphors We Live By, en trad. fr. (1986) Les métaphores dans la vie quotidienne mettant en avant des « configurations mentales».

23. Avec en commun (voir ma note 8) les contraintes papier et/ou écran qui imposent des conventions de notations abrégées, ici 20 caractères pour les formules de LVF sur son support électronique initial, ouvrant vers des consultations "actives» par tris croisés. Cette situation 
devient caduque quand le dictionnaire électronique aménage une possibilité de restituer à volonté les termes abrégés dans leur intégralité.

24. De ce point de vue une place intéressante a été donnée à l'annexe grammaticale très détaillée (développée sur 67 pages) du Dictionnaire Larousse du française langue étrangère niveau 2 (1979) dirigé par Jean Dubois avec Françoise Dubois-Charlier comme première collaboratrice. On y a vu, à juste titre, un jalon vers LVF dans la recherche de critères de classification syntaxiques et surtout sémantiques. En revanche, on n'a pas examiné de près les procédés d'exemplification (commentés en présentation $\mathrm{p}$. VII à X), radicalement différents d'un recours systématique à la phrase simple telle qu'elle est pratiquée dans LVF. Approfondir cette question serait intéressant pour affiner l'analyse des rapports entre apprentissages scolaires élémentaires et présentation scientifique des bases de la grammaire française et de son lexique, en rapport explicite ici avec le «français fondamental » de Georges Gougenheim ([1956] 1967). On sait que cet ouvrage novateur au $20^{\mathrm{e}_{\mathrm{eme}}}$ siècle fut réalisé à l'ENS de Saint-Cloud, dont Jean Dubois aurait souhaité prendre la direction. On y travaillait, au BEL/BELC, les problématiques de l'enseignement du français langue étrangère (futur FLE), favorables à la grammaire raisonnée dès lors qu'on s'inscrit dans un contexte institutionnalisé de traductions entre langues nationales où joue le français en francophonie. Ce contexte, qui apparente l'Algérie francophone au Québec, est explicite dans l'intitulé de ce dictionnaire pour enfants, initialement commandité après 1962 à Jean Dubois quand il effectuait des missions de coopération dans le Laboratoire de Phonétique de l'université d'Alger. Il m'a d'ailleurs appris par la suite au détour d'une conversation que le projet avait achoppé sur le choix des noms propres dans les phrases d'exemples du dictionnaire, Larousse ayant refusé l'alternative, actuellement dépassée, du type de Omar/Pierre va à l'école, présente dans les livres algériens mais dévolue antérieurement aux manuels pour indigènes durant la période coloniale (Achour 1985). Une information intéressante, dans une optique d'histoire de la francophonie et de ses antécédents coloniaux.

25. Dont il n'est jamais assuré qu'ils prévaudront parmi d'autres ouvrages et manuels concurrents, comme l'a bien analysé Sonia Branca (1980 : 54-56) étudiant l'ellipse et l'anaphore chez Condillac et concluant sur les « raisons d'un échec ».

26. En rupture avec de nouvelles habitudes au $21^{\text {ème }}$ siècle qui est enclin à remplacer des multilinguismes, incluant le latin savant, par des partenariats directs entre langues vivantes. Voir sur ce point le DEC et ses clarifications sur terminologies, notions et ontologies dans une optique de formation scolaire et universitaire (Tremblay et Polguère 2014) qui donne la priorité à un bilinguisme orienté de l'anglais au français, sans trancher clairement sur l'orientation inverse français/anglais. Pour une profondeur de champ, sur ces questions de rapports de langues, je renvoie à Lusignan (2004) qui examine le triangle historique latin/français/anglais.

27. Je relève que ces philologues linguistes sont d'ailleurs cités comme des interlocuteurs privilégiés dans la correspondance de Bally étudiée par Curea. Voir également à ce propos comment Chevalier (1994) situe l'héritage composite de Ferdinand Brunot qu'il juge hâtif et erroné de condamner en bloc au nom des sciences du langage.

28. Un exemple typique : le site rattaché au journal Le Monde qui se nomme Dicocitations.

29. Pour bégayer ajoutons cet exemple tiré du journal Marianne (mars 2018) : L'histoire ne se répète pas, elle bégaie, assurait Marx. En ce qui concerne la SNCF, elle paraît franchement radoter...

30. Un exemple d'actualité : l'oxymore sorti des vieilleries rhétoriques qui permet une alliance des contraires dans des objectif culturels de "déconstruction partagée » valorisant les perspectives du désordre dans l'esthétique contemporaine.

31. Mon propos converge avec des remarques de A. Lehmann (1985a, art. cit., p. 118) posées en termes de « littérarité » à partir de Gérard Genette (1991) : «L'objectif de la lexicographie n'est [...] pas de cultiver la fonction esthétique de la citation littéraire, même si elle ne doit pas être totalement sacrifiée [...] En outre - et cela resterait à élucider - il semble que les critères de la littérarité ne sont pas identiques à ceux qui caractérisent le statut et la fonction de l'exemple 
littéraire dans le dictionnaire». Sur ce point, les a priori du Petit Bon usage étroitement apparentés à l'exemplification lexicographique, sont un champ d'observation intéressant à examiner. Un exemple à étudier : le recul d'André Gide privilégié par Maurice Grevisse.

32. Notamment avec le gout des politiques pour la « petite phrase».

33. Jean Dubois était d'ailleurs un spécialiste de la presse écrite avec sa thèse sur le vocabulaire politique (Dubois 1962), portant précisément sur une période historique couverte par Karl Marx (référencé en page XXIV de présentation), servant ici de jalon au cliché enregistré avec LVF.

34. An introduction to descriptive linguistics, New York: Holt, Rinehart and Winston, 1961, traduction française par Françoise Dubois-Charlier, Introduction à la linguistique, Larousse : Paris, 1967-1975.

35. Notamment "Co-Occurrence and Transformation in Linguistic Structure » de Harris, un article de 1957 dans Language, abondamment réédité, contemporain de Syntactic Structures de Chomsky.

36. Voir sur ce point Balibar-Mrabti (2011b) à propos du Dictionnaire du Français Contemporain annonçant dès 1966 le programme du lexique-grammaire au LADL avec cette affirmation: le lexicographe deviendra un syntacticien.

37. Une préoccupation que LVF partage avec le Dictionnaire des verbes du français actuel (Florea et Fuchs 2010) ou encore le Manuel d'analyse linguistique (G. Gross 2012, ouvr. cit.).

38. Les exemples d'applications retenus étant des fragments de textes scientifiques.

39. Secondé par Françoise Dubois-Charlier qui en aura contrôlé le suivi et la cohérence au prix d'un énorme travail d'accompagnement et de vérification.

40. Soulignons que le terme de « combinatoire lexico-syntaxique » est le choix ultime des Dubois quand ils publient leur dernière contribution sur le vocabulaire de la musique parue en 2010.

41. Remarquons ici que l'utilisation des statistiques en linguistique appliquée et l'analyse des ressources lexicales en réseaux, avec leurs outils spécifiques de présentation, induisent un regard distancié sur la seule linéarité et ses deux axes comme supports d'exposition de la syntaxe. Métalexicographie et/ou dictionnairique?

42. Voir notamment le site menapia. discipline.ac-lille.fr

43. Rappelons que les auteurs soulignent le côté "ouvert", autrement dit "productif», des listages de formes à propos de la sous-classe syntaxique C4a étudiée ici. Même souci dans Locutions en français (Dubois et Dubois Charlier 2004) considéré comme crucial : une précision dont Chevalier fait l'éloge (2010, art. cit.) dans son survol général des travaux ancrés autour du LADL, incluant Françoise Dubois-Charlier et Jean Dubois qu'il s'amuse à qualifier de « plus jeune disciple » de Maurice Gross.

44. En parallèle avec Alain Rey et Josette Rey-Debove dirigeant les dictionnaires Robert. Autour des enjeux de cette nouvelle science du détail, nous assistons à une lutte frontale qui met face à face les détenteurs d'une connaissance de la grammaire en durée longue et les informaticiens de l'industrie logicielle (voir ma section 1). Sont ciblés des choix de société qui se doublent de choix lucratifs et se comparent aux réussites en France de maisons d'édition comme Hachette, Hatier, Larousse, au début de l'instruction pour tous passant par le livre papier Nous en gardons la trace historique, symbolisée par le groupe d'édition Hachette. Même trace, en remontant plus haut, avec la typographie Garamond dont le nom renvoie au tout début de l'imprimerie lyonnaise sur papier (Balibar-Mrabti 2017a).

45. Les sociologues ayant fait valoir que plus on a besoin d'un dictionnaire moins on s'en sert et inversement.

46. Selon des reformulations qu'il est intéressant de rapprocher a contrario du cas des proverbes, initialement abordés comme formes fixes, que Jean-Claude Anscombre $(2005$; 2016) a mis au centre de son œuvre et dont il a analysé en détail les variations qu'il classe dans ses «matrices lexicales ".

47. Tombant rarement dans la faiblesse majeure du procédé : l'exemple forcé. 


\section{RÉSUMÉS}

Cet article présente un point de vue historique d'ordre métalexicographique sur le dictionnaire Les verbes français (LVF) de Jean Dubois et Françoise Dubois-Charlier. A partir du tirage papier réalisé en 1997 et destiné aux chercheurs, sont analysés ici les savoir-faire qui président à la confection de l'illustration systématique des entrées verbales. Ce sont des phrases que les habitudes pédagogiques et les nomenclatures officielles désignent en français contemporain comme des " phrases simples ». On examine ici un cas d'emploi métaphorique dans la classe $\mathrm{C}$ des verbes de communication, notamment les formules d'opérateurs qui entrent en jeu au seuil des traitements automatiques proposés. Cet examen demande qu'on s'interroge sur la façon dont LVF extrait et forge ses exemples phrastiques. A travers le filtre sociolinguistique des manuels et des dictionnaires de langue générale, l'ouvrage présente une caractéristique qui mérite analyse : le remaniement, courant chez les lexicographes, de certaines phrases initiales, authentifiées par des signatures d'écrivains. A l'ère de la documentation automatique pour tous, dans sa pratique avertie de l'énoncé bref en durée longue, associant phrases simples et phrases forgées, le dictionnaire Les verbes français aura brillé pour s'assurer l'adhésion de ses utilisateurs, spécialistes et non spécialistes, quant aux règles grammaticales et sémantiques de la phrase dite libre du français écrit actuel.

How does the search for exemplification work with regard to sentences which are built with the purpose of illustrating verbs used in a metaphorical acception? The answer here is based on the examination of Jean Dubois and Françoise Dubois-Charlier's class C verbs, 'verbes de communication', and presents a type of inheritance running through various cases of written usage. The examples are extracted from historical successions of dictionaries of the past, especially the Larousse series. Contemporary as well as traditional examples, extracted from newspapers and grammar exercises as well as exercises in style taught in textbooks, are also used. All of them need to be reformulated into anonymous 'simple sentences'. Through their judicious choices of simple sentences, the authors of Les verbes français succeed in convincing the reader that there can be no discussion about the judgments of acceptability, in what they define as current modern speech in French.

\section{INDEX}

Mots-clés : métalexicographie, phrase simple, métaphore, cliché, littératie

Keywords : metalexicography, simple sentence, metaphor, cliché, literacy

\section{AUTEUR}

\section{ANTOINETTE BALIBAR-MRABTI}

Université de Picardie Jules Verne 